\title{
An Adaptive IEEE 802.11 MAC in Multihop Wireless Ad Hoc Networks Considering Large Interference Range*
}

\author{
Tzu-Chieh Tsai and Chien-Ming Tu \\ Department of Computer Science \\ National Chengchi University \\ \{ttsai, g8912\}@es.nccu.edu.tw
}

\begin{abstract}
The IEEE 802.11 standard is the most popular Medium Access Control (MAC) protocol for wireless local area networks. However, in multihop wireless ad hoc networks, the IEEE 802.11 MAC protocol will suffer from more serious hidden terminal and exposed terminal problems than those in single hop WLANs. More specifically, it is due to the "large" interference range and the "large" carrier sensing range. In this paper, we focus on the collisions caused by the existence of large interference range in multihop wireless ad hoc networks and propose an adaptive IEEE 802.11 MAC (AMAC) that makes two simple modifications of IEEE 802.11 RTS/CTS handshake to dynamically adjust the transmission and reception according to the shared medium status near transmitter and receiver, respectively. Simulation results show that our method can lessen interferences and increase system throughput as compared with IEEE 802.11 MAC in the multihop wireless ad hoc networks.
\end{abstract}

\section{Introduction}

People have been dreaming to communicate with anyone, anytime, anywhere. With the recent advances in wireless technologies and the development of mobile computing devices, it is now possible and popular to build high-speed wireless systems that are easy to install and operate. Wireless is the only medium that can facilitate such communications.

A wireless ad hoc network is a collection of mobile nodes equipped with wireless transceivers that form an autonomous network without the help of any fixed networking infrastructure. A node can transmit data packets directly to other nodes which are within its radio coverage range or via multihop store-and-forward relay to nodes outside the range. Such network received considerable attention in recent years in both commercial and military applications due to its attractive properties of building a network on the fly without requiring any pre-planned infrastructure such as base stations or a central controller. These factors make the study of multihop ad hoc networks very interesting. This paper will focus on issues of multihop networks.

* This work was supported under NTPO project NSC91-2219-004-004

R. Battiti, M. Conti, and R. Lo Cigno (Eds.): WONS 2004, LNCS 2928, pp. 87-100, 2004.

(C) IFIP International Federation for Information Processing 2004 
Because the wireless is a broadcast medium, it is inevitable that multiple devices access medium at the same time, thus results in garbled data so-called collision. And, the frequency is a scarce resource and is a shared medium. Efficiently controlled access of this shared media becomes a complicated and important task. Therefore, many medium access control (MAC) protocols were developed [1][2]. Among all theses standards, e.g. IEEE 802.11, HIPERLAN1/2, Bluetooth; the IEEE 802.11 is the most popular protocol used in both WLANs and MANETs (Mobile Ad-hoc Networks).

In this paper, we study interference problems resulted from large interference range when the IEEE 802.11 MAC is used in multihop wireless ad hoc networks and then propose two modifications of the RTS/CTS handshake in the IEEE 802.11 MAC. The large interference range means that the range of interference is larger than the transmission range. In the past, most researches assumed that hidden terminals are located inside the transmission range, few of them considered the large interference range, which is very serious in multihop wireless ad hoc networks.

The rest of this paper is organized as follows. In Section 2 we review the IEEE 802.11 MAC protocol, hidden terminal and exposed terminal problems. In Section 3 we explain the large interference range and its influences. Then we present our proposed modifications in detail including transmitter side control and receiver side control in Section 4. Simulation results using NCTUns network simulator are given in Section 5 and we then conclude this paper in Section 6.

\section{Background}

The IEEE 802.11 MAC protocol [3][4] defines two different access method, one is the fundamental mechanism to access the medium called distributed coordination function (DCF), another is an optional point coordination function (PCF), is a centralized MAC protocol able to support collision free and time bounded services. Because the PCF is not suitable for distributed ad hoc networks, we now describe the DCF in detail.

\subsection{The DCF of the IEEE 802.11 MAC Protocol}

The DCF is also known as carrier sense multiple access with collision avoidance (CSMA/CA). For a mobile node to transmit, it shall sense the medium to determine if another mobile node is transmitting. If the medium is not determined to be busy for greater than or equal to a DIFS (DCF IFS) period, the transmission may proceed. If the medium is determined to be busy, the mobile node shall defer until the end of the current transmission. After deferral, or prior to attempting to transmit again immediately after a successful transmission, the mobile node shall select a random backoff interval and shall decrement the backoff interval counter while the medium is idle. Whenever the backoff timer reaches zero, transmission shall commence. 


\subsection{The Hidden Terminal and Exposed Terminal Problems}

The CSMA/CA mechanism was designed to avoid collisions, however, resulted in the hidden terminal problem [5][6], and exposed terminal problem [6].

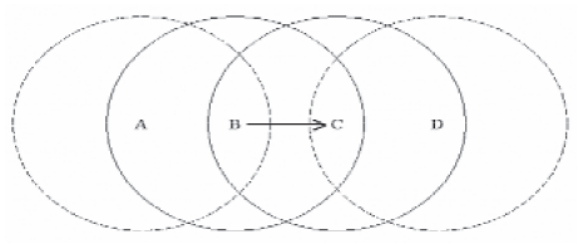

Fig. 1. Hidden terminal and exposed terminal.

A hidden terminal is one that is within the range of the intended receiver but out of range of transmitter. Consider the case shown in Figure 1. Station B is transmitting to station C. Station D cannot hear the transmission from B. During this transmission when D senses the channel, it falsely thinks that the channel is idle. If station D starts a transmission, it interferes with the data reception at $C$. In this case station $D$ is a hidden terminal to station B. Hence, hidden terminals can cause collisions on data transmission.

Exposed terminals are complementary to hidden terminals. An exposed terminal is one that is within the range of the transmitter but out of range of the receiver. In Figure 1, station A can hear the transmission from B. When station A senses the channel, it thinks that the channel is busy. However, any transmission by station A does not reach $\mathrm{C}$, and hence does not interfere with data reception at station $\mathrm{C}$. In this case, station $\mathrm{A}$ is an exposed terminal to station B. Ideally, station A can send simultaneously to other receivers without interfering C's reception. Therefore, if expected terminals are prohibited to transmit, it will underutilize available bandwidth.

\subsection{The RTS/CTS Handshake in IEEE 802.11 MAC}

The main task of a MAC is to avoid collisions. In order to eliminate hidden terminal problems, the IEEE 802.11 MAC protocol defines an optional four-way handshaking technique, known as request-to-send/clear-to-send (RTS/CTS) mechanism. A mobile node that wants to transmit, follows rules explained in Section 2.1, and then, instead of the data frame, preliminarily transmits a special short frame called request to send (RTS) to reserve the channel. When the destined node receive the RTS frame, it responds, after a SIFS, with a clear to send (CTS) frame. The transmitting node is allowed to transmit its frame only if the CTS frame is correctly received. All other nodes that hear either the RTS and/or the CTS set their virtual CS indicator, called a network allocation vector (NAV), for the given duration indicated in the RTS/CTS frame. The NAV state is combined with physical carrier sense function to indicate the busy state of the medium. This mechanism reduces the probability of the receiver side collision caused by a node that is hidden from the transmitter. Therefore, all other 
nodes inside the transmission range of transmitter/receiver that hear the RTS/CTS will defer their transmission and thus avoid collisions caused by hidden terminals.

\section{Large Interference Range}

In recent years, more and more researchers have realized large interference range [7][8][9]. The large interference range means that the range of interference is larger than the transmission range as following.

\subsection{The Large Interference Range}

Considering the signal propagation, some nodes that are out of the transmission range of both the transmitter and the receiver, may still interfere with the receiver. This situation happened rarely in the singlehop WLAN because almost all the mobile nodes are within each other's transmission range. But in the multihop wireless ad hoc networks, the phenomenon does exist and becomes a serious problem. To prove this fact, [7] uses a simple analytic model to show that in the open space environment, the interference range of a receiver is 1.78 times the transmitter-receiver distance as Figure 2 shows. That is, if the distance between the transmitter, $S$, and receiver, $R$, is $d$, then the interference range of receiver is $1.78 * \mathrm{~d}$, which may be larger than the transmission range. Any stations inside this interference range once transmitting to other nodes can interfere R's reception. This result overthrows the early assumption of interfering nodes are within the transmission rang.

\subsection{The Large Interference Range in the NS-2 Simulator}

There is also another thing needed to be noticed that the carrier sense wireless networks are engineered in such a way that the carrier sensing and interference range is typically larger than the range at which receivers are willing to accept a packet from that same transmitter [10]. Many researchers use the famous NS-2 network simulator from Lawrence Berkeley National Laboratory (LBNL) [11] with extensions from the MONARCH project at Carnegie Mellon [12] to estimate their proposals. These extensions include a set of mobile ad hoc network routing protocols and an implementation of BSD's ARP protocol, as well as an IEEE 802.11 MAC protocol.

In the NS-2 network simulator, the interfering range (and sensing range) is larger than the communication range. It is implemented using a simple BER model: if the received power level of the incoming frame below the carrier sense threshold, the frame is discarded as noise; if the received power level is above the carrier sense threshold but below the receive threshold, the frame is passed to the MAC layer but marked as a packet in error; otherwise, the frame is simply forwarded up to the MAC layer. 
In the IEEE 802.11 MAC of NS-2, when a mobile node is receiving a frame, and some other frame is also transmitted during its reception, two cases could happen. If the power level of the packet already being received is at least $10 \mathrm{~dB}$ greater than the received power level of the new packet, the MAC layer assumes "capture", discards the new packet, and allows the receiving interface to continue with its current receive operation; otherwise, a collision occurs and both frames are dropped. Here the $10 \mathrm{~dB}$ is a typical value in network simulator while modeling the receiver's capability to capture signal from noise. As shown in Figure 3, a collision happens when a signal propagated inside carrier sense range arrives earlier than a signal propagated inside a transmission range arrives. Because the later stronger signal collides the formal weak signal receiving. This is also an instance of large interference range.

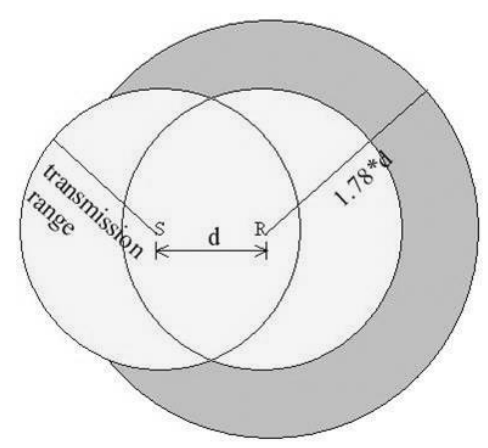

Fig. 2. Large interference range

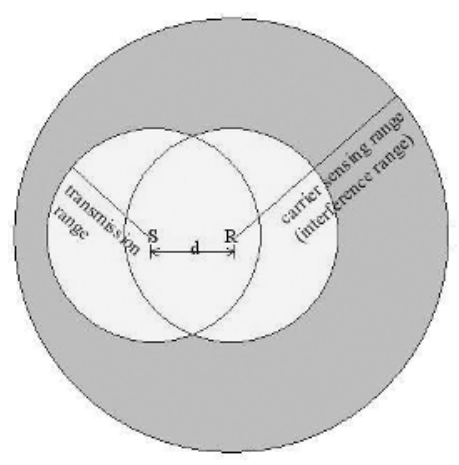

Fig. 3. Large interferences range in NS-2

\subsection{The Influences of Large Interference Range}

The RTS/CTS handshake was proposed to solve the hidden terminal problem based on an assumption that hidden nodes are within transmission range of the receiver. With overhearing the CTS control frame, the hidden terminals near the receiver can be inhibited so that the RTS/CTS can eliminate most of interference.

Ideally, the RTS/CTS handshake can eliminate most of interference. However, if the large interference range is concerned, the RTS/CTS is not so effective. Nodes located outside the transmission range of both transmitter and receiver may still interrupt reception [7], even if the RTS/CTS is supported. Because nodes located outside transmission range of transmitter and/or receiver cannot receive the RTS/CTS correctly, they won't keep silent if they happen to have something to transmit. Moreover, the large interference range will cause more collisions either on control frames or data frames. This results in more serious problems such as TCP instability and unfairness [8]. This situation is infrequent in an 802.11 basic service set, because all nodes can sense each other's transmissions. However, in an ad hoc network, it becomes a serious problem due to the large distribution of mobile nodes and the multihop operation. 
In [7], they propose a simple MAC layer scheme called Conservative CTS Reply (CCR). An intended receiver only replies a CTS frame to a RTS initiator when the receiving power of that RTS frame is larger than a certain threshold (CTS_REPLY_THRES-HOLD). This CTS_REPLY_THRES-HOLD should be larger than the threshold required for a node to successfully receive a packet. For example, let $\mathrm{R}_{\mathrm{tx}}$ denotes the transmission range. The value is chosen as a receiving power at a receiver which is $0.56 * \mathrm{R}_{\mathrm{tx}}$ away from the transmitter. Since when the transmitterreceiver distance is smaller than $0.56 * \mathrm{R}_{\mathrm{t} x}$, the interference range is smaller than $1.78 *$ $\left(0.56 \mathrm{R}_{\mathrm{tx}}\right)$, which is $\mathrm{R}_{\mathrm{tx}}$. So the whole interference area is covered by RTS/CTS handshake. Therefore it can totally eliminate the collisions caused by large interference range. The illustration of transmission range, interference range, and CTS reply range are shown in Figure 4, where the CTS reply range is the range of a receiver willing to reply CTS back to the RTS initiator.

However, the CCR is over conservative that limits the available radio utilization range. Only transmitters inside $0.56 * \mathrm{R}_{\mathrm{tx}}$ of receiver are allowed to transmit, and the available transmission area is reduced to $31.36 \%$ (i.e. $0.56^{2}$ ) of physical transmission area, even if there are no hidden terminals in the interference range. Moreover, it is not effective if mobile nodes are sparse or the traffic is not heavy.

We propose an Adaptive IEEE 802.11 MAC (AMAC) that makes two simple modifications of the RTS/CTS handshake to reduce the influences of collisions resulted from large interference range in the multihop wireless ad hoc networks.

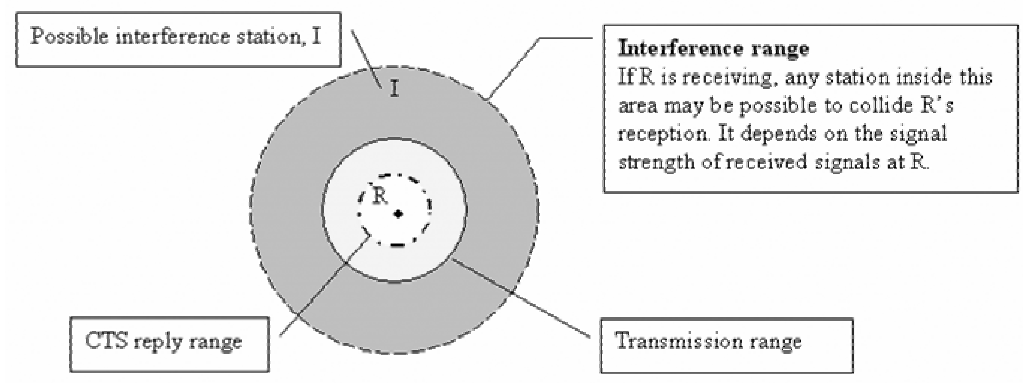

Fig. 4. The illustration of transmission range, interference range, and CTS reply range.

\section{The AMAC Mechanism}

The AMAC (Adaptive MAC) protocol modifies the IEEE 802.11 RTS/CTS handshake on transmitter (RTS) and receiver (CTS), respectively. The principle of our modification is "What you wish done to yourself, do to others; what you do not wish done to yourself, do not do to others." In reception phase, we make the hardware's best to receive but estimate for the probability of successful reception to prevent from 
useless weak signal transmission (compared to hidden terminals' signal) which could be collided with hidden terminals' transmissions near the receiver. In transmission phase, we not only consider the most transmission opportunity but also give neighboring nodes chances to transmit or receive.

\subsection{The Receiver Side Control Mechanism}

Inspired from the CCR scheme, we propose a simple mechanism that dynamically adjusts the value of "CTS_REPLY_THRESHOLD" according to the historical neighboring medium usage status. If the receiver has some information about the interfering nodes, it can estimate the interference probability, and set the appropriate CTS_REPLY_THRESHOLD. Note that the CCR scheme assigns the CTS reply range to be a fixed value, for example $0.56 * \mathrm{R}_{\mathrm{tx}}$, while our goal is aimed to dynamically adapt the CTS reply range according to the channel status at receiver side.

Mobile nodes periodically sense the medium status and record the sensed signal strength as shown in Figure 5. Let mobile nodes sense the medium status every sense_signal_interval micro seconds, total number of sensing is sense_times. The sensed signal strength will be recorded and the oldest recorded information will not be kept if the number of sensed records exceeds sense_times. Note that we only record the sensed signal strength transmitted inside the interference range but outside the receiving range, i.e. the signal transmitted inside the gray area of Figure 4. The recorded signal strength will be used to compute the CTS_REPLY_THRESHOLD, which concerns the potential transmissions that can cause interferences.

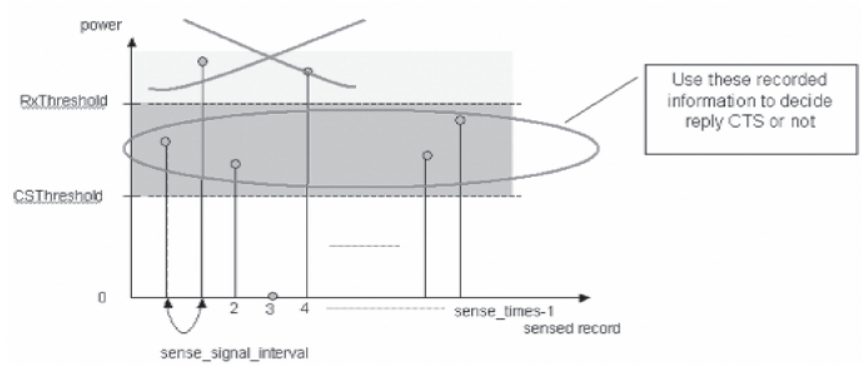

Fig. 5. Sense the medium periodically.

The considerations of CTS reply include two limitations. One is the estimation of whether interference will happen or not; another is the probability of interference if it happens. With the first limitation, we calculate the average of recorded signal strengths not equal to zero as equation (1), where $\Delta_{i}$ is used to identify whether $P_{i}$ is needed to be included or not; $n$ is the sense_times; $P_{i}$ is the sensed signal strength between the threshold of receiving and of carrier sensing, and the Capture_Threshold is the capability the receiver can capture signal from noise. Usually the Cap- 
ture_Threshold is 10. Hence, the CTS_REPLY_THRESHOLD could reflect average interference status.

$$
\begin{aligned}
& \Delta_{i}=\left\{\begin{array}{l}
1, \text { carrier_sense_threshold } \leq P_{i}<\text { receive_threshold } \\
0, \text { otherwise }
\end{array}\right. \\
& \text { CTS _REPLY_THRESHOLD }=\left(\frac{\sum_{i=0}^{n-1} P_{i} * \Delta_{i}}{\sum_{i=0}^{n-1} \Delta_{i}}\right) * \text { Capture_Threshold }
\end{aligned}
$$

With the second limitation, we consider the probability of the reception be collided $\left(\mathrm{p}_{\text {collided }}\right)$. Again we use the past recorded signal strength. This probability is obtained as equation (2), where $\Delta_{i}$ is used to identify whether $P_{i}$ larger than $P_{R T S}$ or not; the $P_{R T S}$ is the received signal strength of the RTS control frame.

$$
\begin{aligned}
& \Delta_{i}= \begin{cases}1, & P_{i}>P_{R T S} \\
0, & \text { otherwise }\end{cases} \\
& p_{\text {collided }}=\frac{\substack{n-1 \\
\sum \Delta_{i}}}{n}
\end{aligned}
$$

After receiving RTS successfully, the intended receiver first checks if the power of the received RTS is larger than the computed CTS_REPLY_THRESHOLD; if larger, then he replies CTS back; if not larger, then he reply CTS with probability $1-p_{\text {collided }}$.

This is because collisions happen at receivers. It is better for receivers to take the responsibility for monitoring the signal statuses and to estimate admitting receptions. So, if a node $\mathrm{I}$, which is located outside the transmission range of receiver $\mathrm{R}$ and transmits frequently, any transmitter $\mathrm{S}$ may experience a collision at $\mathrm{R}$, even if the $\mathrm{RTS} / \mathrm{CTS}$ handshake is presented. If $\mathrm{R}$ decides to only reply $\mathrm{S}$ when the receiving signal strength of $\mathrm{S}$ is higher than the calculated CTS_REPLY_THRESHOLD, derived from R's observation, the interference will be reduced. On the other hand, if neighboring nodes transmit infrequently, then the CTS_REPLY_THRESHOLD should not be hold as the CCR specified; the threshold should be relaxed. This can be done by our periodical computation so as to dynamically reflect the current situation.

This mechanism has two benefits. One is that it can adapt to surrounding channel status. If the channel is always busy or the channel is too noisy around the receiver, then the receiver will decide not to reply the CTS for a demand RTS request. Another benefit is that although the receiver may not reply a CTS back to the RTS requestor, it is still a good decision because the "weak" data transmission (compared to the stronger interference signal strength at receiver) may not be received by the receiver, due to large interference range. At the same time, this weak data transmission could still collide other nodes' receptions near the transmitter, just like the transmitter's data collided by other nodes at receiver. So this mechanism should be beneficial to wireless ad hoc networks. 


\subsection{The Transmitter Side Control Mechanism}

As Section 2 stated, the CSMA/CA, which is a random access control mechanism, is contention-based. All mobile nodes have to contend for the channel to transmit. As explained before, due to large interference range, a transmission may be collided by another transmission and collide other's transmission. There is a need to control unnecessary or excessive transmission, so we propose the transmitter side control mechanism that adapts to neighborhood situations. And also could improve some degree of fairness.

Before actually sending out a control frame, the RTS, the mobile node first checks the neighbors' activities, which is gotten from the periodically sensing the receiver side does it. Let $\delta_{i}$ denote whether neighboring nodes transmit at $\mathrm{i}^{\text {th }}$ carrier sensing or not; $N$ is the total number of neighboring nodes' transmission activities; and neighbor_tx_ratio is defined as the portion the total number of times neighboring nodes transmit in sense_times periodically sense as equation (3) shows.

$$
\begin{aligned}
& \delta_{i}= \begin{cases}1, & P_{i}>\text { carrier_sense_threshold } \\
0, & \text { otherwise }\end{cases} \\
& N=\sum_{i=0}^{n-1} \delta_{i} \\
& \text { neighbor_tx_ratio }=\frac{N}{n}
\end{aligned}
$$

If neighbor_tx_ratio is less than a neighbor_tx_threshold, and the mobile node himself sent recently, this may imply that neighboring nodes hardly contend for the channel. So we force the mobile node to backoff to release the medium access for neighboring nodes.

Another case for this is that the neighboring nodes' silence is because of no data to send or being inhibited by RTS/CTS. So after backoff, mobile node observes whether the total number of neighboring nodes' transmission activities increases or not. If yes, the neighbor_tx_threshold is increased. Otherwise, it may imply that the neighboring nodes really have no data to send out, and the mobile node can speed up its transmission. In this case, the neighbor_tx_threshold is decreased. Here we use heuristic method to change the value of neighbor_tx_threshold.

By our both receiver side and transmitter side AMAC control, we can reduce fragile frame that the power strength of receiving signal at receiver is low or easily be collided with hidden terminals in the interference range, by not responding CTS frame, and won't collide with other's reception if the node transmits. We can also achieve MAC level fairness in some degrees by means of our transmitter side control to give other nodes chances to transmit or to receive. 


\section{Performance Evaluation}

We use the NCTUns simulator [13] to justify our proposed modification of the IEEE 802.11 MAC protocol. The MAC protocol of the NCTUns is ported from NS-2 network simulator which implements the complete IEEE 802.11 standard MAC protocol DCF to accurately model the contention of nodes for the wireless medium. All nodes communicate with identical, half duplex, wireless radios that are modeled after the commercially available 802.11(b)-based Wave-Lan wireless radios, which have a bandwidth of $11 \mathrm{Mb} / \mathrm{s}$ and a nominal transmission range of 250 meters with the carrier sensing range is $550 \mathrm{~m}$. Note here that the value of CTS_REPLY_THRESHOLD is chosen as a receiving power at a receiver which is $0.56 * \mathrm{R}_{\mathrm{tx}}$ away from the transmitter in the following simulations.

\subsection{The Simulation of 1 TCP Flow via Multihop Transmission}

We first setup a simple simulation to analyze the relationship between the interferences and the distance of transmitter-receiver. Each node is identical. The path loss model is set to Two-Ray Ground model; DSDV routing protocol is used; the traffic generator is a greedy TCP. The topology of this simulation is a 7 nodes chain topology as shown in Figure 6.

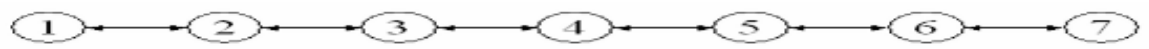

Fig. 6. The topology of 1 TCP flow simulation.

The distance between any two neighboring nodes is equal, let $\mathrm{d}(\mathrm{i}, \mathrm{i}+1)$ be the distance between node $i$ and node $i+1$. We adjust the distance of $d(i, i+1)$ from $100 \mathrm{~m}$ to $200 \mathrm{~m}$ to observe the throughput variation. As the distance between each node increases, the number of hops increases. The simulation result is shown in Figure 7.

We can see that when $d(i, i+1)$ is less than $125 \mathrm{~m}$, the throughput of IEEE 802.11 is high. This is because with such distances, the transmission from node 1 to node 7 only involves 3 hops and each sender, i.e. the node 1 , node 3 , and node 5 can sense each other, therefore can avoid most interferences. However, once the distance $d(i, i+1)$ is bigger than $125 \mathrm{~m}$ (i.e. more than 3 hops from node 1 to 7 ), the throughput degrades significantly. This confirms that the IEEE 802.11 was primordially designed for WLAN, was not designed for multihop ad hoc network, the IEEE 802.11 MAC cannot function well in such networks.

We also take a look of the CCR scheme, the throughput was not good in this scenario, it is because the CCR is over conservative that cannot reflect the neighboring nodes' situations so that they only accept the received signal strength of RTS control frame greater than Conservative CTS_REPLY_THRESHOLD. With d(i, i+1) larger than $141 \mathrm{~m}(250 \mathrm{~m} * 0.56)$, the CCR won't reply CTS. Hence, no transmissions occur.

Now, let us consider our proposed scheme, AMAC. The throughput is not good in contrary to IEEE 802.11 MAC protocol when the $\mathrm{d}(\mathrm{i}, \mathrm{i}+1)$ is $100 \mathrm{~m}$ and $125 \mathrm{~m}$. This is 
because our control in transmitter and receiver will reduce the transmission rights. However, when the distance is larger, the performance of AMAC is better than the other two MAC protocols. This is because our scheme can decrease the probability of collisions. As a metric define in [7], we define the "data frame collision ratio" as the portion of data frames transmitted at the MAC layer which are collided at the intended receiver due to collision; the retransmission data frame is viewed as a new data frame. We also only count the data frame collision ratio of unicast data frames, thus exclude routing packets that are broadcasting based. The simulation result of data frame collision ratio is presented in Figure 8.

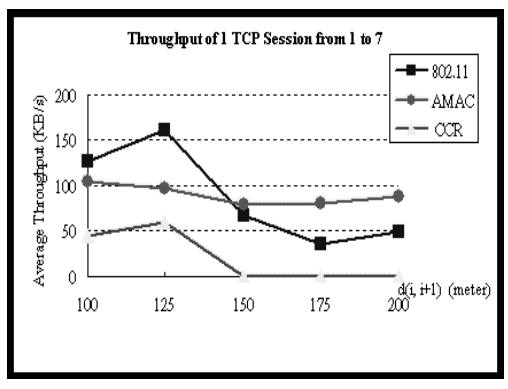

Fig. 7. The data frame collision ratio of 1 TCP session from node 1 to node 7 .

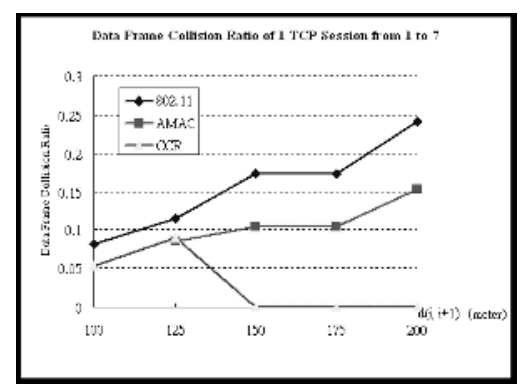

Fig. 8. The throughput of 1 TCP session from node 1 to node 7 .

From Figure 8, we can see that the IEEE 802.11 suffers from more serious colliding than CCR and AMAC. It is also a hint that the contention manner of CSMA/CA may not suit to multihop connections. Every node constructive to compete the medium interferes other nodes. Eventually, every node interferes every node. Therefore, it is evidenced that the current version IEEE 802.11 not function well in multihop wireless ad hoc networks. There should have some efforts to overcome collision problems resulted from large interference range.

\subsection{The TCP Instability Problem Simulation}

In [8], they revealed the TCP instability problem in IEEE 802.11 multihop networks. This problem is resulted from the insufficient access control of IEEE 802.11 MAC. Here we redo the same simulation as [8] stated. The simulation topology is still a chain topology as Figure 6 shown with d(i, i+1) is $200 \mathrm{~m}$. The parameters are also the same as section 5.1 used. The traffic generator is a TCP sender that always has data to send out. DSR routing protocol is used. The traffic is from node 1 to node 5 . The simulation result is shown in Figure 9.

From the figure, we can see that our proposed scheme can eliminate the TCP instability problem. The reason is that the senders controlled by our AMAC adjust their transmission rate, which can fit well with this chain multihop transmission. 


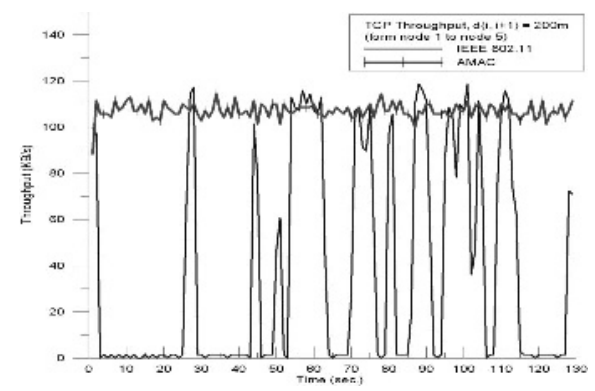

Fig. 9. The TCP throughput.

\subsection{The Large Interference Range Simulation}

We now estimate the influences of large interference range. The simulation topology is shown in Figure 10. We let the distance d(i, i+1) 200m, except d(2, 3) is well calculated to 355 meters so that $\mathrm{d}(1,3)$ is 555 meter that node 3 and node 1 cannot sense each other, but node 3 can interfere node 2 . CCR is not applicable here.
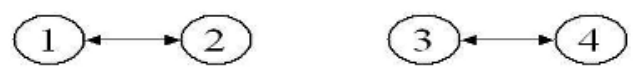

Fig. 10. The topology of interference simulation.

The scenario is that node 1 sends CBR/UDP traffic to node 2 as "Flow 1", the CBR packet size is 1024 bytes and the packet rate is 500pps, node 3 sends VBR/UDP traffic to node 4 as "Flow 2", where the VBR packet size is also 1024 bytes (Poisson dist.). VBR packet rate is increasing by time to observe how it interferes with flow 1.

\subsection{Random Topology Simulation}

Finally, we simulate in a random topology scenario. 100 nodes are randomly placed in a $1000 \mathrm{~m} * 1000 \mathrm{~m}$ area. DSR routing protocol is used. We randomly select 4 sourcedestination pairs to send UDP/CBR traffic. The packet rate is 500 pps with packet size 1024 bytes. 

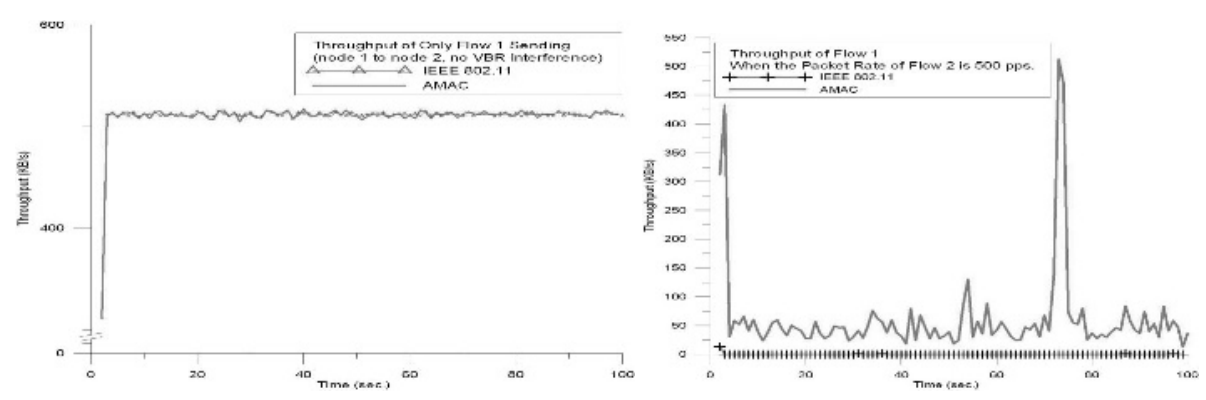

Fig. 11. The throughput of only node 1 sending.
Fig. 12. The throughput comparison when the packet rate of node 3 is 500 pps.

The simulation results are shown in Figure 13 and Figure 14. We can see that the throughput of AMAC is higher than the IEEE 802.11 and CCR. This is because our scheme can reduce the collisions, thus improve the throughput. The CCR scheme can really eliminate the data corruption, but the throughput is not very good because it needs more hops to reach destination.

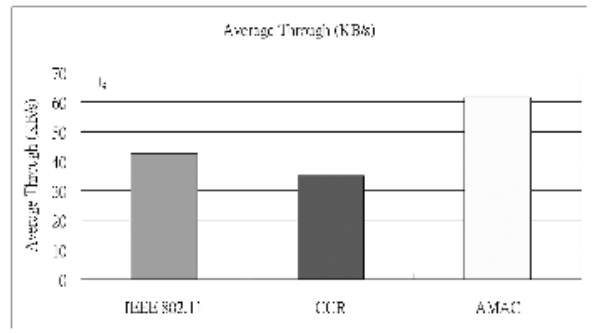

Fig. 13. throughput for the random topology.

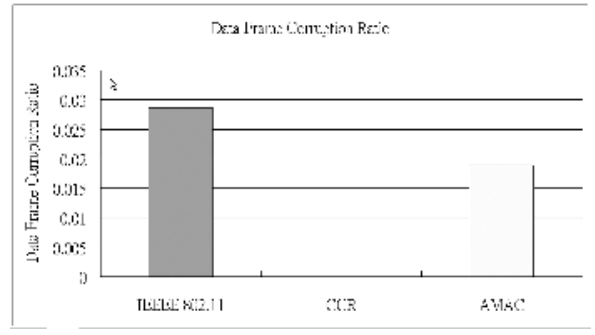

Fig. 14. Data frame corruption ratio.

\section{Conclusion}

In the multihop wireless ad hoc networks, the performance of IEEE 802.11 MAC degrades dramatically. The networks suffer from more serious hidden terminal problem than the WLAN because of large interference range. In this paper we are inspired from previous analysis of [7] to propose an adaptive MAC (AMAC) scheme by modifying the IEEE 802.11 MAC RTS/CTS handshake. We add two control mechanisms on transmitter and receiver with the objective of reducing probability of collisions and reducing the number of collisions. The simulation results show that our modification used in the multihop wireless ad hoc networks outperforms the IEEE 802.11 MAC. Besides, there are some issues for future work, for example the estimation model at 
receiver may work well and the optimum value of parameters used in both transmitter side and receiver side.

\section{References}

1. Antoine Mercier, Pascale Minet, Laurent George, and Gilles Mercier, "Adequacy between multimedia application requirements and wireless protocols features," IEEE Wireless Communications, vol.9 No.6, pp. 26-34, December 2002.

2. Ramiro Jordan and Chaouki T. Abdallah, "Wireless communications and networking: an overview," IEEE Antenna's and Propagation Magazine, vol. 44 no. 1, February 2002.

3. IEEE, 1999, Wireless LAN Media Access Control (MAC) and Physical Layer (PHY) Specifications, IEEE Std. 802.11.

4. Crow, B.P.; Widjaja, I.; Kim, L.G.; Sakai, P.T., "IEEE 802.11 Wireless Local Area Networks," IEEE Communications Magazine, Volume: 35 Issue: 9, pp.116-126, Sept. 1997.

5. L. Kleinrock and F. Tobagi, "Packet switching in radio channels, part II-the hidden terminal problem in carrier sense multiple access and the busy tone solution," IEEE Trans. Commun., vol. COM-23, no. 12, pp. 1417-1433, Dec. 1975.

6. P. Karn, "MACA-A New Channel Access Method for Packet Radio," in Proc. 9th ARRL Computer networking Conference, 1990.

7. Kaixin $\mathrm{Xu}$, Mario Gerla, and Sang Bae, "How effective is the IEEE 802.11 RTS/CTS handshake in ad hoc networks?" GLOBECOM 2002 - IEEE Global Telecommunications Conference, no. 1, pp. 72-77, November 2002.

8. Xu, S.Saadawi, T. "Does the IEEE 802.11 MAC Protocol Work Well in Multihop Wireless Ad Hoc Networks?" IEEE Communications Magazine, Volume: 39 Issue: 6, pp. 130-137, June 2001.

9. Jinyang. Li, Charles Blake, Douglas S. J. De Couto, Hu Imm Lee, and Robert Morris, "Capacity of ad-hoc wireless networks, “ Proceedings of ACM MOBICOM 01, pp. 61-69, July 2000 .

10. Joao L. Sobrinho, A. S. Krishnakumar, "Quality-of-Service in Ad Hoc Carrier Sense Multiple Access Wireless Networks," IEEE Journal on Selected Areas in Communications, no. 8, pp. 1353-1368, August 1999.

11. VINT Group, UCB/LBNL/VINT network simulator-ns (version 2), http://www.isi.edu/nsnam/ns.

12. The CMU Monarch Project, Wireless and mobility extension to ns, http://www.monarch.cs.cmu.edu.

13. S.Y. Wang, C.L. Chou, C.H. Huang, C.C. Hwang, Z.M. Yang, C.C. Chiou, and C.C. Lin, "The Design and Implementation of the NCTUns 1.0 Network Simulator", Computer Networks, Vol. 42, Issue 2, pp. 175-197, June 2003. 